\title{
Free-breathing 3D phase-sensitive inversion recovery late gadolinium enhancement at 3.0 Tesla: reliability and image quality in ischemic and non-ischemic cardiomyopathy in comparison with multiple breath-hold 3D imaging
}

\author{
Maurice Bizino*, Jacob Amersfoort, Qian Tao, Rob J van der Geest, Hildo J Lamb
}

From 18th Annual SCMR Scientific Sessions

Nice, France. 4-7 February 2015

\section{Background}

In both ischemic (ICM) and non-ischemic (NICM) cardiomyopathy late gadolinium enhancement (LGE) is an important cardiovascular magnetic resonance (CMR) technique. LGE CMR, traditionally performed in 2D during multiple breath-holds (MB), is challenging for vulnerable patients and subject to slice misregistration. Therefore, LGE CMR during free-breathing $(\mathrm{FB})$ is more robust and enables to perform 3D acquisition, increase resolution and apply phase-sensitive inversion recovery (PSIR). We developed and clinically tested a high spatial resolution PSIR LGE sequence using a respiratory navigator in both ICM and NICM patients. Reliability and image quality were compared to a multiple breath-hold 3D approach (MB-3D).

\section{Methods}

Approval was waived by the institutional ethical committee. Between May 2012 and November 2013 FB-3D and MB-3D sequences were acquired in patients suspected of ICM and NICM. Data of 48 patients $(36$ men; mean age \pm standard deviation: 60.8 years $\pm 10.9 ; 34$ ICM) were retrospectively collected. LGE sequences were acquired using a 3.0 Tesla MR system. For both FB-3D $(1.68 \times 1.68 \times 3.4 \mathrm{~mm})$ and MB-3D $(1.86 \times 2.8 \times$ $10 \mathrm{~mm})$ a PSIR sequence was used. Multiplanar reformats with high resolution (FB-3D-HR; $0.91 \mathrm{~mm}$ isotropic voxels) and low resolution (FB-3D-LR; $1.86 \times 2.8 \times$ $10 \mathrm{~mm}$ ) were constructed. LGE mass and image quality
(SNR, CNR and edge sharpness) were compared using the Friedman test, Wilcoxon signed rank test, spearman correlation and Bland-Altman analysis.

\section{Results}

34 ICM patients (23 chronic MI; 4 sub-acute MI; 7 acute MI) and 14 NICM were included (5 dilated cardiomyopathy; 4 hypertrophic cardiomyopathy; 3 peri-/ myocarditis; 2 other). In figures 1 and $23 \mathrm{D}$ reconstructions of two patients are shown.In the complete dataset of 48 patients, there were no significant differences between FB-3D-HR, FB-3D-LR and MB-3D datasets in terms of LGE mass (FB-3D-HR: (median [interquartile range]): $8.5 \mathrm{~g}$ [4.4-19.9]; FB-3D-LR: $10.7 \mathrm{~g}$ [4.5-22.9]; MB-3D: $9.8 \mathrm{~g}$ [5.1-19.8]; $\mathrm{p}=0.099)$. LGE mass correlated well (FB-3D-HR vs MB-3D: $r=0.922, p=0.01$; FB3D-LR vs MB3D: $r=0.922, p=0.01$ ) with Bland Altman analysis indicating good agreement for FB-3D-HR vs MB-3D (mean difference \pm SD: $1.15 \pm 5.45$ gram) and FB-3D-LR vs MB-3D (mean difference \pm SD: $0.00 \pm$ 5.47 gram). SNR was higher in both FB-3D-HR (median [interquartile range]: 244 [192-326], $\mathrm{p}=0.001)$ and FB-3D-LR (825 [498-1417], $\mathrm{P}<0.001)$ as compared to MB-3D (200 [146-263]). CNR was also higher in both FB-3D-HR (226 [164-277], $\mathrm{p}<0.001)$ and FB-3D-LR (691 [480-1086], $\mathrm{p}<0.001)$ as compared to MB-3D (216 [162260]). Edge sharpness was $25 \%$ higher in FB-3D-HR as compared to MB-3D (0.21 [0.19-0.23] vs 0.17 [0.15$0.20], \mathrm{p}<0.001)$. 

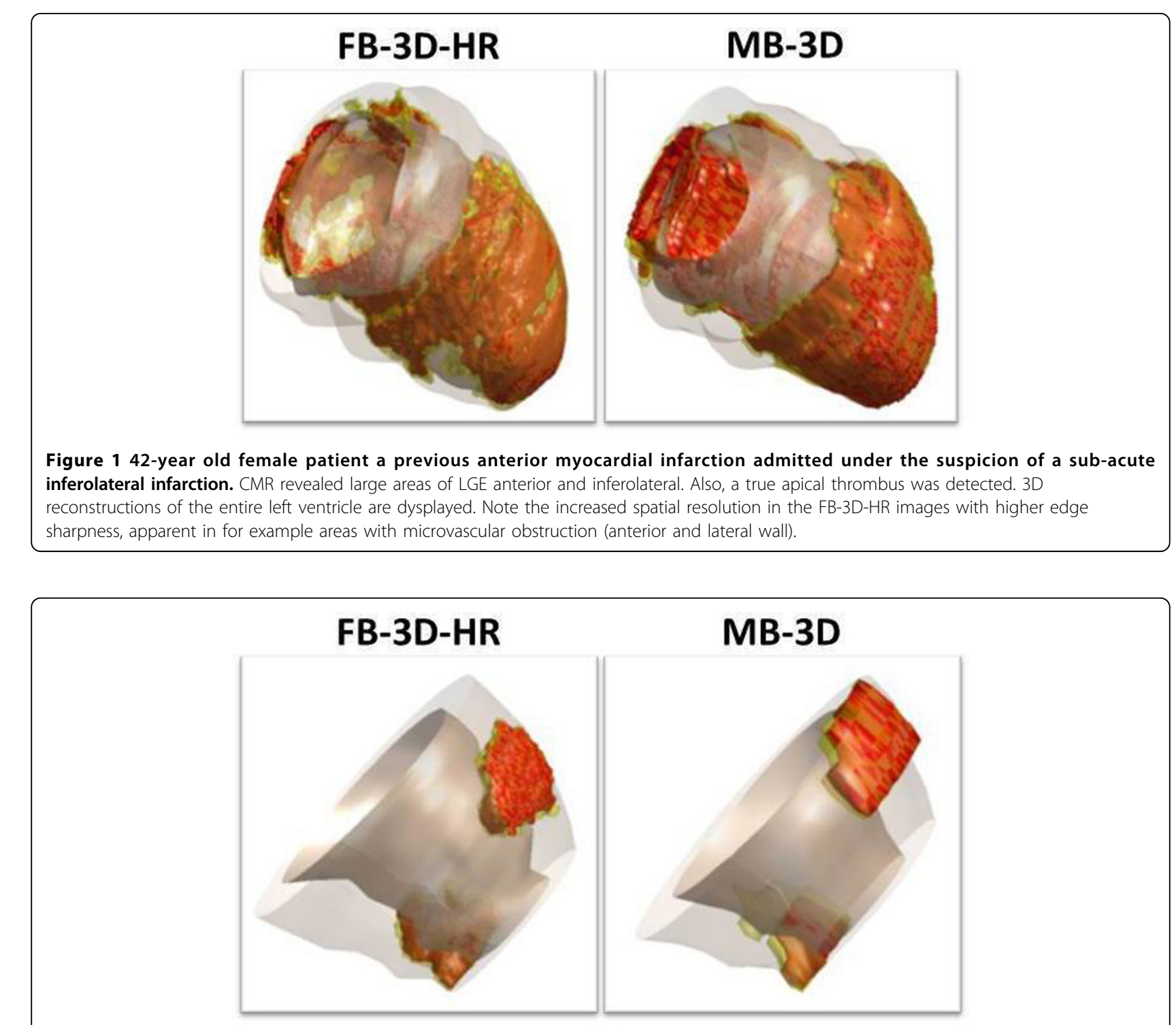

Figure 2 49-year old male patient admitted with thoracic pain related to breathing with elevated serum creatine kinase and troponine values. CMR reveals LGE in the epicardial apico-anterior wall and extending into the pericardium, and in the epicardial apico-inferior wall suggesting perimyocarditis. Appreciate the high contrast in the FB-3D images and sharp delineation of LGE areas in the FB-3D-HR 3D reconstruction.

\section{Conclusions}

Free-breathing 3D phase-sensitive inversion recovery LGE CMR enables reliable myocardial scar tissue assessment with significantly improved image quality as compared with multiple breath-hold 3D imaging in ischemic and non-ischemic cardiomyopathy.
doi:10.1186/1532-429X-17-S1-P97

Cite this article as: Bizino et al:: Free-breathing 3D phase-sensitive inversion recovery late gadolinium enhancement at 3.0 Tesla: reliability and image quality in ischemic and non-ischemic cardiomyopathy in comparison with multiple breath-hold 3D imaging. Journal of Cardiovascular Magnetic Resonance 2015 17(Suppl 1):P97.

\section{Funding}

No funding. 